\title{
Cronoterapia dos fármacos anti-hipertensores: uma revisão baseada na evidência
}

Ângela Oliveira Mendes, ${ }^{1}$ Filipa Oliveira Pinheiro ${ }^{2}$

\section{RESUMO}

Objetivo: Determinar se a administração noturna de fármacos anti-hipertensores poderá ter benefício em termos de eficácia na redução dos valores tensionais e/ou de eventos cardiovasculares, morbilidade e mortalidade comparativamente à sua administração no período matinal.

Fontes de dados: National Guideline Clearinghouse, NHS Evidence, CMA Infobase, The Cochrane Library, DARE e MEDLINE/PubMed.

Método de revisão: Pesquisa de guidelines, revisões sistemáticas (RS), meta-análises (MA) e ensaios clínicos publicados entre janeiro de 2008 e outubro de 2018, disponíveis nas línguas inglesa e portuguesa, com os termos MeSH Antihypertensive Agents e Drug Chronotherapy. Foi aplicada a escala Strength of Recommendation Taxonomy (SORT), da American Family Physician, para avaliação dos níveis de evidência (NE) e da força de recomendação (FR).

Resultados: Obtiveram-se 55 artigos na pesquisa inicial, dos quais 13 cumpriram os critérios de inclusão. Com a administração no período noturno dos fármacos verificou-se essencialmente um maior controlo dos valores tensionais no período noturno e reversão de perfis não dipper a dipper, sem diferenças estatisticamente significativas no período diurno. Apenas a MA analisou a mortalidade global e cardiovascular, não tendo sido detetadas alterações estatisticamente significativas neste parâmetro entre os grupos de administração no período matinal e noturno; no entanto, foi evidente em três dos estudos um menor risco relativo de eventos cardiovasculares no grupo de administração no período noturno.

Conclusão: A administração de fármacos anti-hipertensores no período noturno comprovou ter benefício em termos de redução dos valores tensionais e mostrou superioridade nessa redução durante o período noturno, permitindo inclusivamente reverter padrões não-dipper/riser a dipper. No entanto, são necessários estudos posteriores, com uma maior uniformidade de critérios e avaliação da morbimortalidade $\mathrm{CV}$, de forma a garantir a eficácia e segurança da administração noturna de fármacos anti-hipertensores.

Palavras-chave: Cronoterapia; Anti-hipertensores.

\section{INTRODUÇÃO}

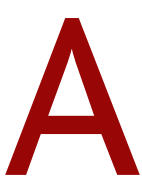
hipertensão arterial (HTA) consiste na elevação persistente dos valores de tensão arterial (TA), definindo-se, em consultório, como um valor de pressão arterial sistólica (PAS) igual ou superior a 140mmHg e/ou de pressão arterial diastólica (PAD) igual ou superior a $90 \mathrm{mmHg}{ }^{1}$

A TA apresenta uma variação circadiana frequentemente caracterizada por um aumento no período matinal, seguida de um ligeiro declínio no período pós-prandial e de uma redução ainda mais evidente no pe-

1. Médica Interna de Medicina Geral e Familiar. USF São Lourenço.

2. Médica Interna de Medicina Geral e Familiar. UCSP Resende. ríodo noturno. Em utentes saudáveis, a TA sofre um decréscimo de cerca de $10-20 \%$ durante o período noturno (perfil dipper); pacientes com uma queda noturna inferior a $10 \%$ são definidos como não dipper e aqueles que apresentam um aumento paradoxal dos valores tensionais neste período designam-se como risers ou dippers invertidos. ${ }^{2}$ Os padrões não dipper e riser constituem importantes fatores de risco para o desenvolvimento de hipertrofia ventricular esquerda (HVE), microalbuminúria, doença cerebrovascular, insuficiência cardíaca congestiva (ICC) e enfarte do miocárdio. ${ }^{3}$

O controlo dos valores tensionais com recurso a fármacos anti-hipertensores, associados a estilos de vida 
saudáveis quando estes não são suficientes, é crucial para a redução da morbimortalidade cardiovascular e global associada à doença. ${ }^{4}$ A medicação anti-hipertensora é tipicamente administrada no período matinal; porém, o momento de ingestão destes fármacos pode afetar o ritmo circadiano da tensão arterial, ${ }^{5-6}$ tendo já sido identificados benefícios em termos de redução dos valores tensionais com a administração de fármacos anti-hipertensores no período noturno. ${ }^{3} \mathrm{Na}$ base destas diferenças encontra-se a possibilidade de reversão de perfis não dipper e riser para dipper com a toma noturna da medicação.?

Face à posologia habitual dos fármacos anti-hipertensores no período matinal e à crescente evidência dos benefícios da sua administração no período noturno, esta revisão pretende determinar se a administração noturna de fármacos anti-hipertensores poderá ter benefício em termos de redução dos valores tensionais e/ou de eventos cardiovasculares, morbilidade e mortalidade comparativamente à sua administração no período matinal.

\section{MÉTODOS}

Foi realizada uma pesquisa de guidelines, revisões sistemáticas (RS), meta-análises (MA) e ensaios clínicos aleatorizados (ECA), utilizando os termos MeSH Antihypertensive Agents e Drug Chronotherapy nas bases de dados National Guideline Clearinghouse, NHS Evidence, CMA Infobase, The Cochrane Library, DARE e MEDLINE/PubMed. Foram incluídos os artigos publicados entre janeiro de 2008 e outubro de 2018, escritos nas línguas portuguesa ou inglesa, que cumprissem os seguintes critérios de eligibilidade:

a) População: Indivíduos de raça não negra com idade $>18$ anos e diagnóstico de hipertensão arterial essencial;

b) Intervenção: Toma de medicação anti-hipertensora no período noturno;

c) Comparação: Toma de medicação anti-hipertensora no período da manhã;

d) Outcome: Controlo dos valores tensionais, eventos $\mathrm{CV}$, morbimortalidade $\mathrm{CV}$.

Foram excluídos os artigos cujo propósito não fosse a avaliação da eficácia da terapêutica a nível tensional e redução de eventos cardiovasculares e/ou morbi- mortalidade, bem como estudos já incluídos nas MA identificadas. Ainda foram excluídos os artigos escritos noutro idioma que não o português ou inglês. Dois dos artigos apenas permitiam a consulta do resumo, tendo sido igualmente excluídos. O processo de seleção dos artigos encontra-se disponível para consulta em fluxograma (Figura 1).

Para avaliação dos níveis de evidência (NE) e da força de recomendação (FR) foi utilizada a escala Strenght of Recommendation Taxonomy (SORT), da American Family Physician.

\section{RESULTADOS}

Da pesquisa bibliográfica inicial obtiveram-se 55 artigos, dos quais apenas 14 cumpriram os critérios de inclusão referidos: duas MAe 12 ensaios clínicos aleatorizados.

A revisão sistemática de Liu e colaboradores ${ }^{2}$ englobou sete estudos experimentais - três ensaios clínicos aleatorizados (ECA) e quatro ensaios clínicos não aleatorizados (ECNA) - e estudou o efeito da administração de pelo menos um fármaco anti-hipertensor no período noturno em pacientes hipertensos com doença renal crónica (DRC). Os investigadores verificaram que, nos ECA, a administração noturna reduziu a TAS e aumentou a TAD na média das $24 \mathrm{~h}$ de forma não significativa (Quadro I); no entanto, tendo em consideração apenas o período noturno, os valores de TAS e TAD apresentaram uma redução estatisticamente significativa $(p<0,0001$ e $p=0,003$, respetivamente), o que não foi verificado no período diurno. A análise dos ECNA não demonstrou alterações estatisticamente significativas nestes parâmetros. Dois estudos demonstraram que a posologia noturna de fármacos anti-hipertensores não contribuiu para a redução da mortalidade global e da morbimortalidade cardiovascular. Em contrapartida, foi objetivado um risco relativo significativamente menor de eventos (enfarte agudo do miocárdio [EAM], angina de peito e revascularização coronária) em ambos os estudos.

A revisão sistemática de Wang e seus colaboradores ${ }^{6}$ incluiu três ECA, dois dos quais já mencionados na revisão de Liu e colaboradores, ${ }^{2}$ e envolveu 3.380 participantes com diagnóstico de HTA e DRC. Foi verificada uma redução significativa da TAS e TAD no período noturno, o que não foi evidente no período das $24 \mathrm{~h}$. Foi objetivado um maior controlo tensional no grupo tratado no período noturno durante os períodos acordado e a 


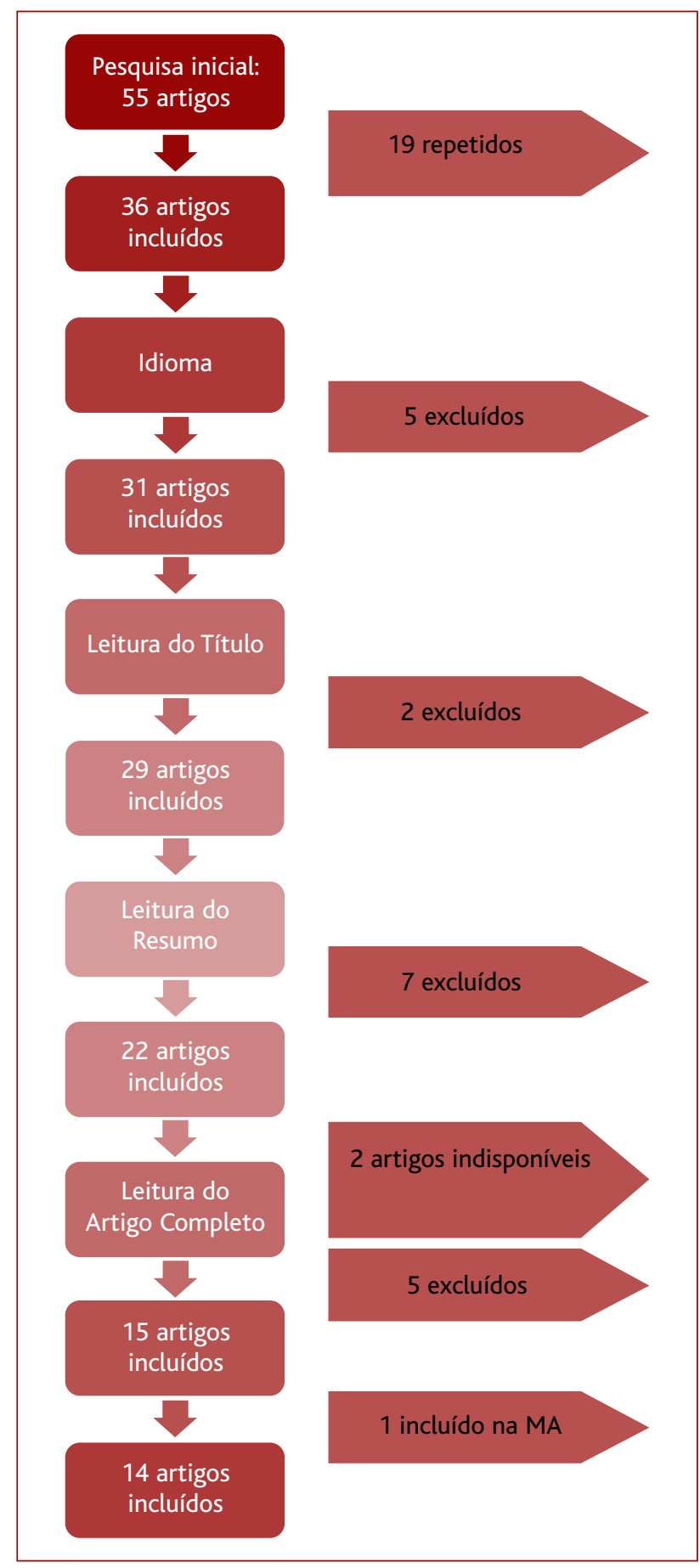

Figura 1. Fluxograma de seleção de artigos incluídos.

dormir, sem diferenças significativas relativamente ao grupo tratado no período matinal. Ainda, um estudo que envolveu a presença de 90 participantes mostrou que em utentes com perfil tensional não dipper, o tratamento com valsartan no período noturno preveniu a deterioração da massa do ventrículo esquerdo (VE), o que não se verificou com a toma matinal do fármaco. Um outro estudo com 2.659 participantes reportou que o índex de rigidez arterial em ambulatório não mostrou diferenças significativas entre grupos.

Em 2009, Hermida e seus colaboradores ${ }^{8}$ procuraram avaliar o efeito da administração de ramipril em monoterapia no período matinal e no período noturno em pacientes com HTA essencial não controlada. Foi realizado um estudo prospetivo, multicêntrico, que envolveu 115 doentes, tendo sido verificada uma redução significativa da média da TA nas $24 \mathrm{~h}$ em ambos os grupos, sendo que no grupo submetido a tratamento no período matinal os efeitos terapêuticos foram mais notórios durante o período acordado, enquanto que nos elementos tratados no período noturno esses efeitos foram mais notórios no período do sono, sendo esta última estatisticamente significativa $(p<0,001)$. Os autores ressalvam que estudos clínicos baseados na monitorização da tensão arterial em ambulatório, realizados em pacientes medicados com ramipril nas dosagens de 5 a $10 \mathrm{mg}$, proporcionavam uma cobertura dos valores tensionais durante $24 \mathrm{~h}$, o que não foi verificado com a dosagem de $2,5 \mathrm{mg}$, sendo a dosagem de fármaco ministrada neste estudo de $5 \mathrm{mg} .{ }^{9}$ Quando o fármaco foi administrado consistentemente no período noturno denotou-se um aumento significativo do rácio da TA no período desperto/período de sono $(p<0,001)$ e uma diminuição significativa quando o fármaco era administrado no período matinal $(p<0,001)$. O perfil tensional noturno dos pacientes também sofreu alterações de acordo com a posologia do fármaco: a proporção de pacientes com perfil não dipper aumentou após a ingestão de ramipril no período matinal $(p=0,001)$ e diminuiu significativamente quando ingerido no período noturno $(p=0,026)$, tendo ainda sido verificada uma redução do surto tensional matinal para aproximadamente metade neste grupo. A percentagem de doentes com controlo tensional em ambulatório foi também superior no grupo tratado no período noturno ( $65 \%$ vs $43 \%$ ).

No ano seguinte, Hermida e seus colaboradores ${ }^{7}$ elaboraram um estudo prospetivo com 203 doentes e demonstraram uma redução significativa do rácio TAS/TAD superior no grupo tratado com uma combi- 


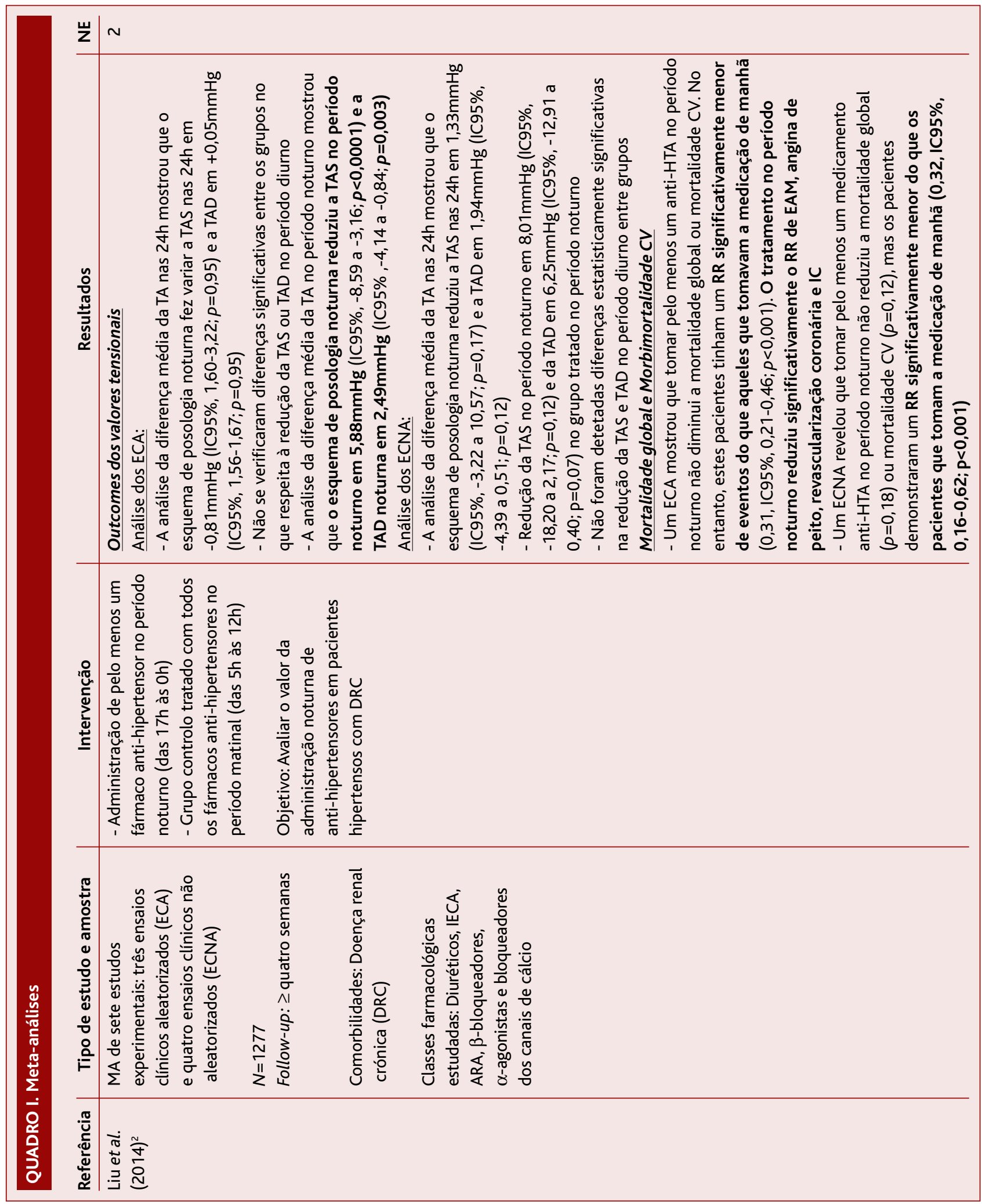




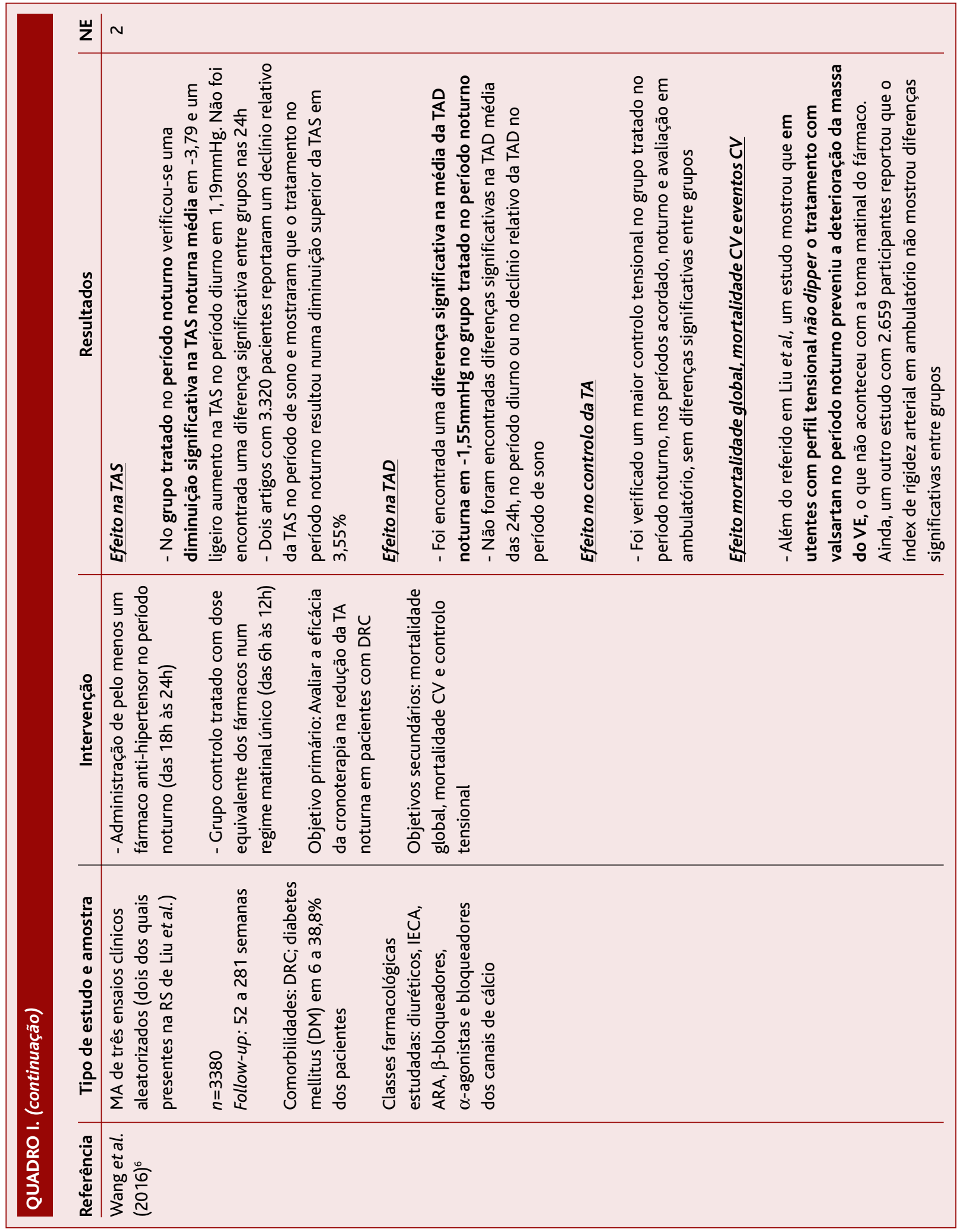




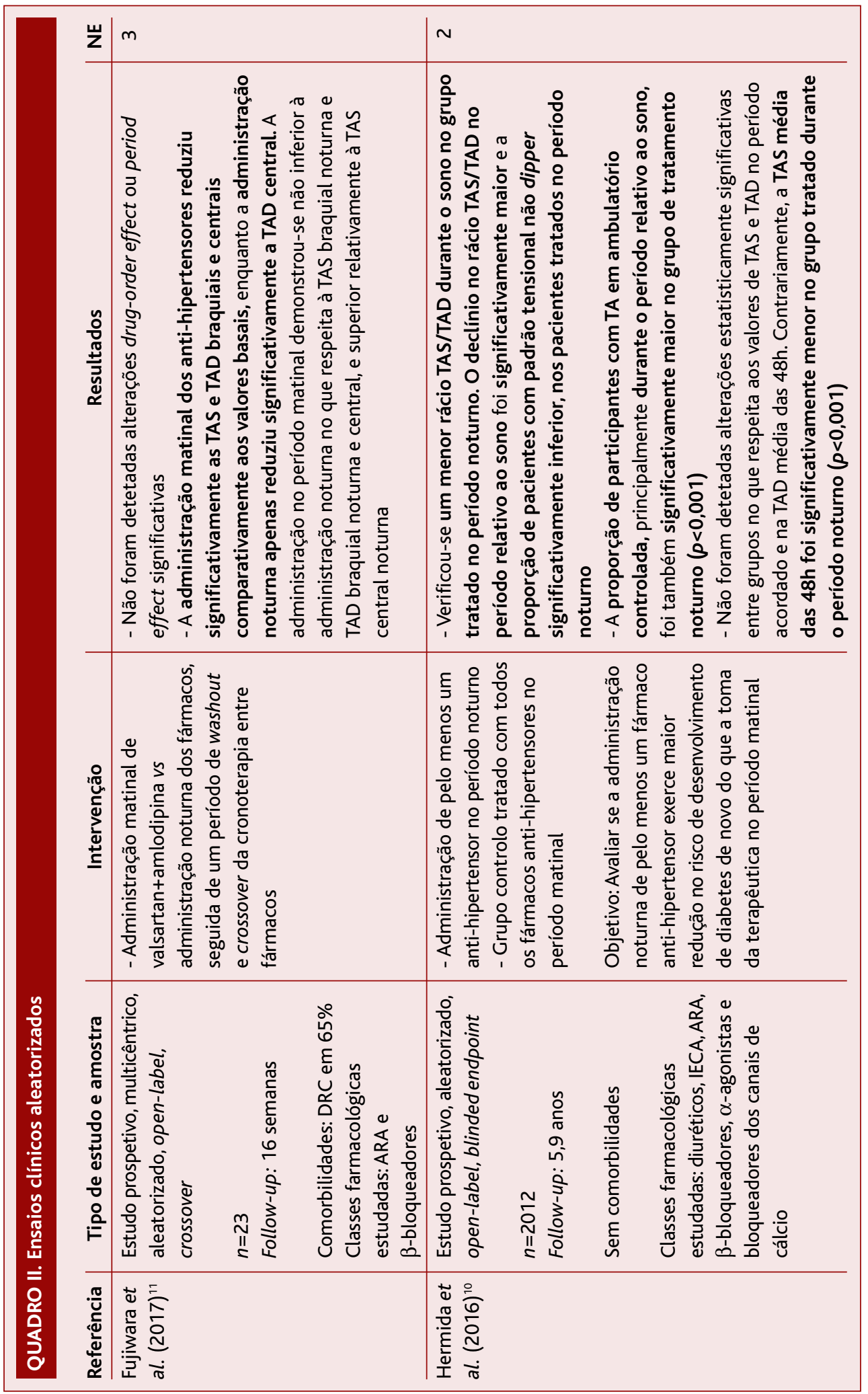




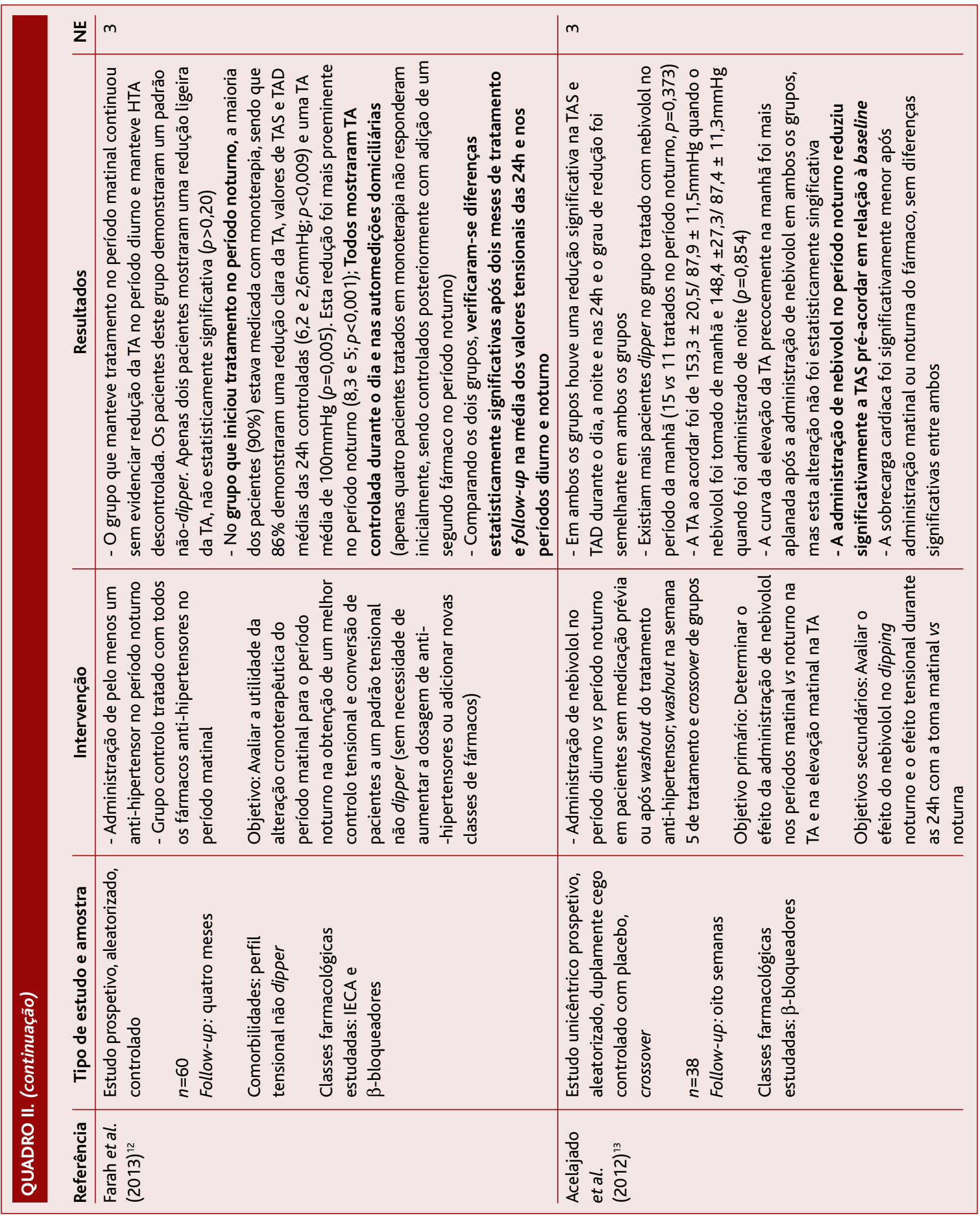




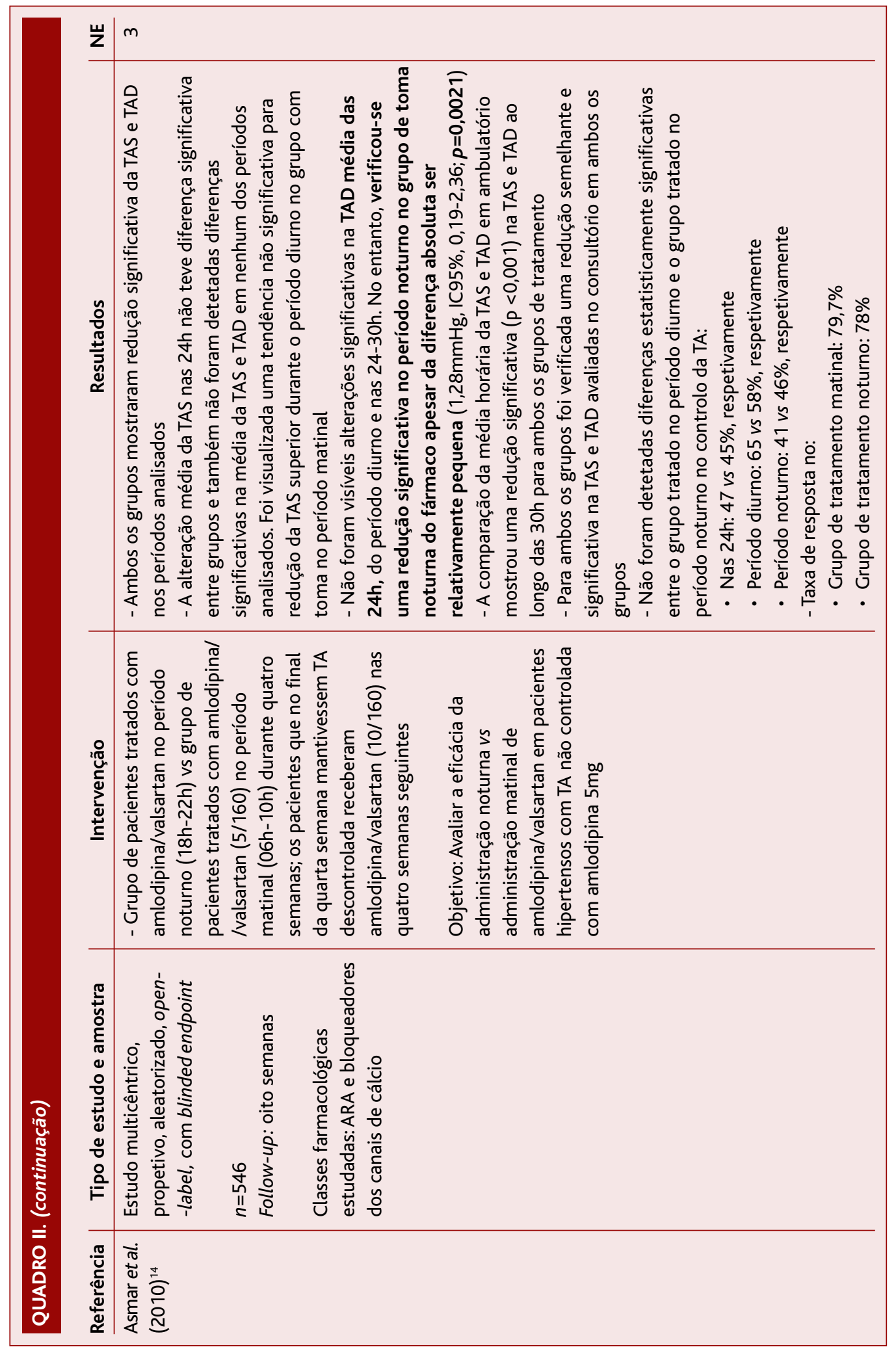




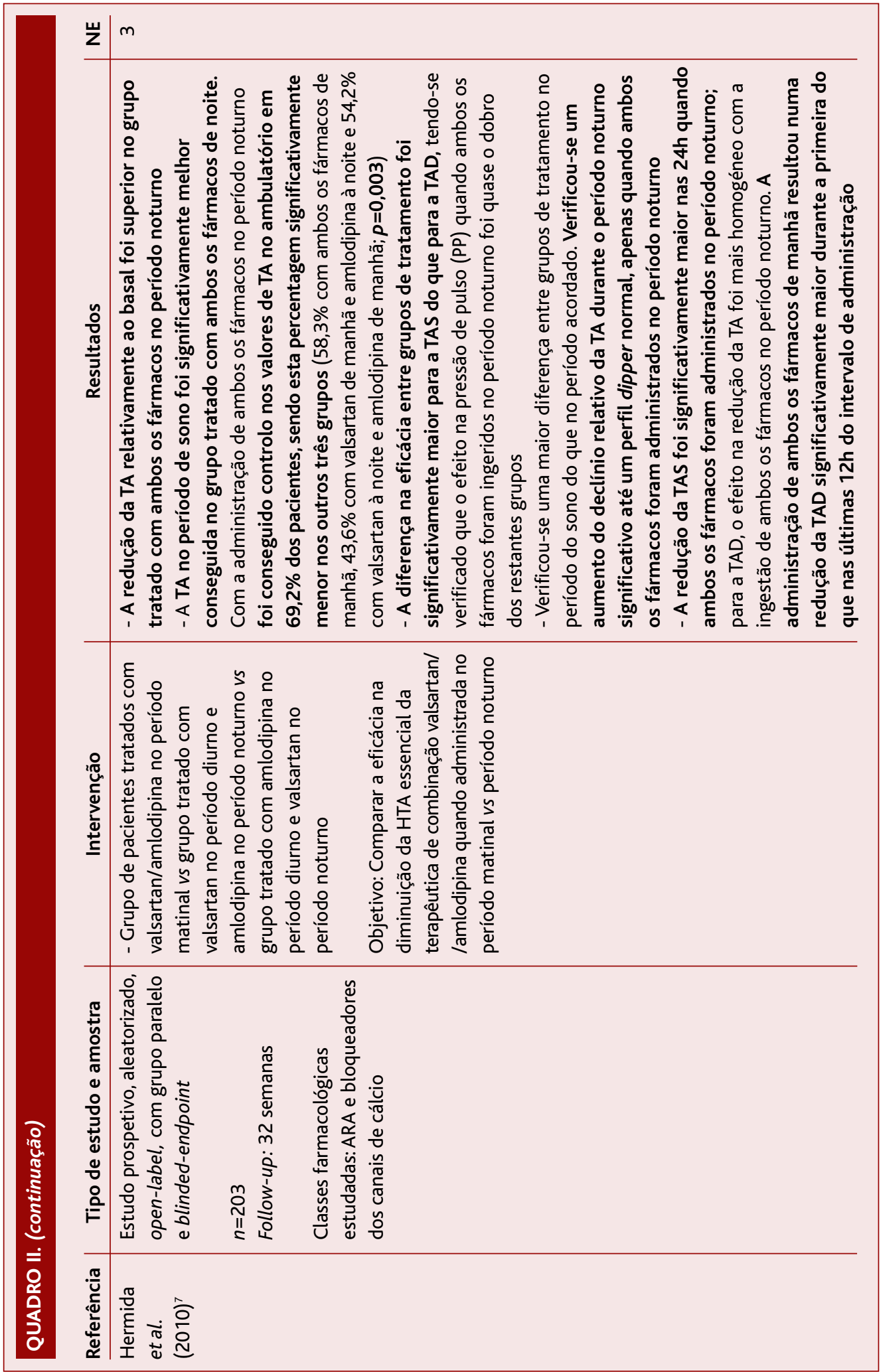




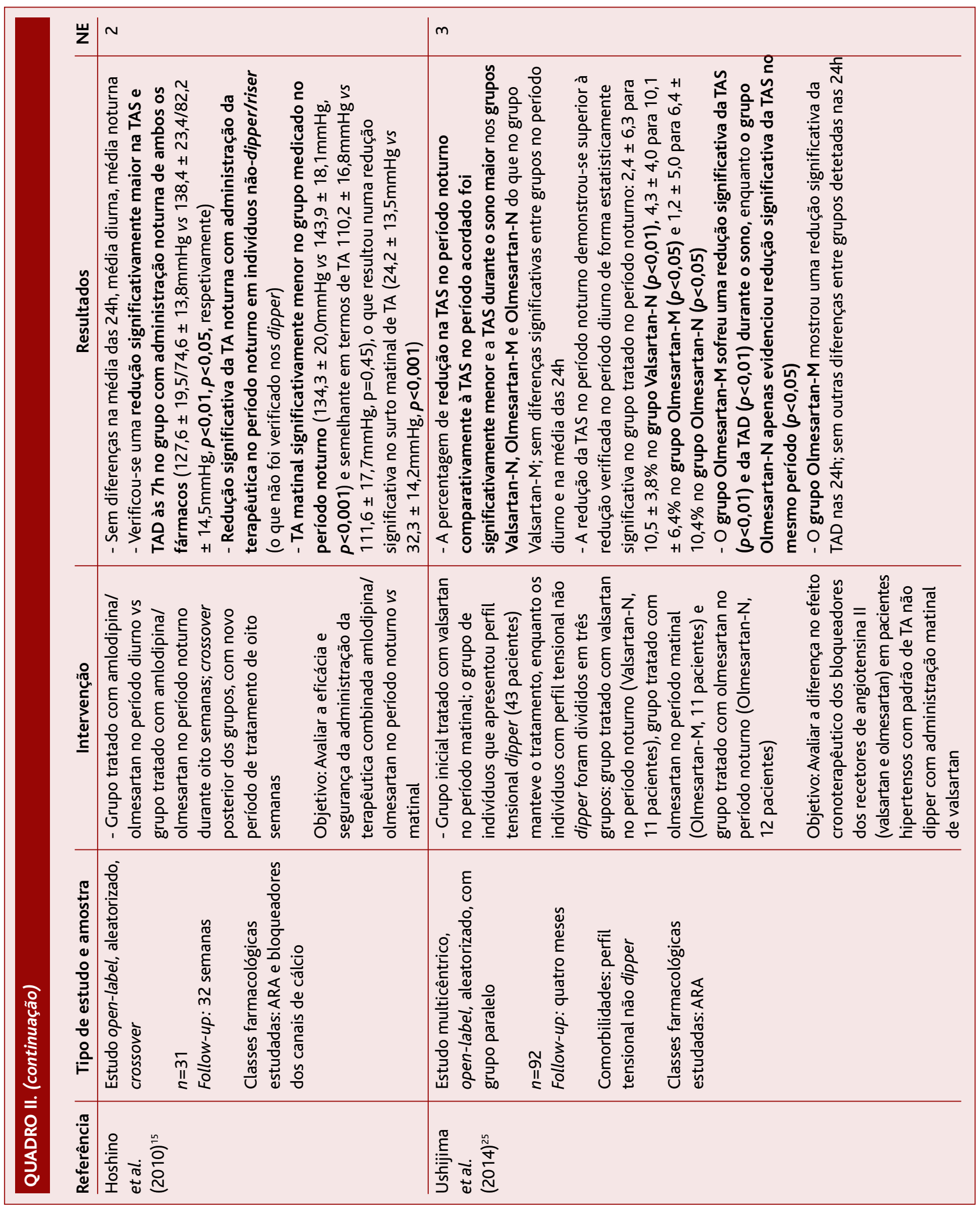




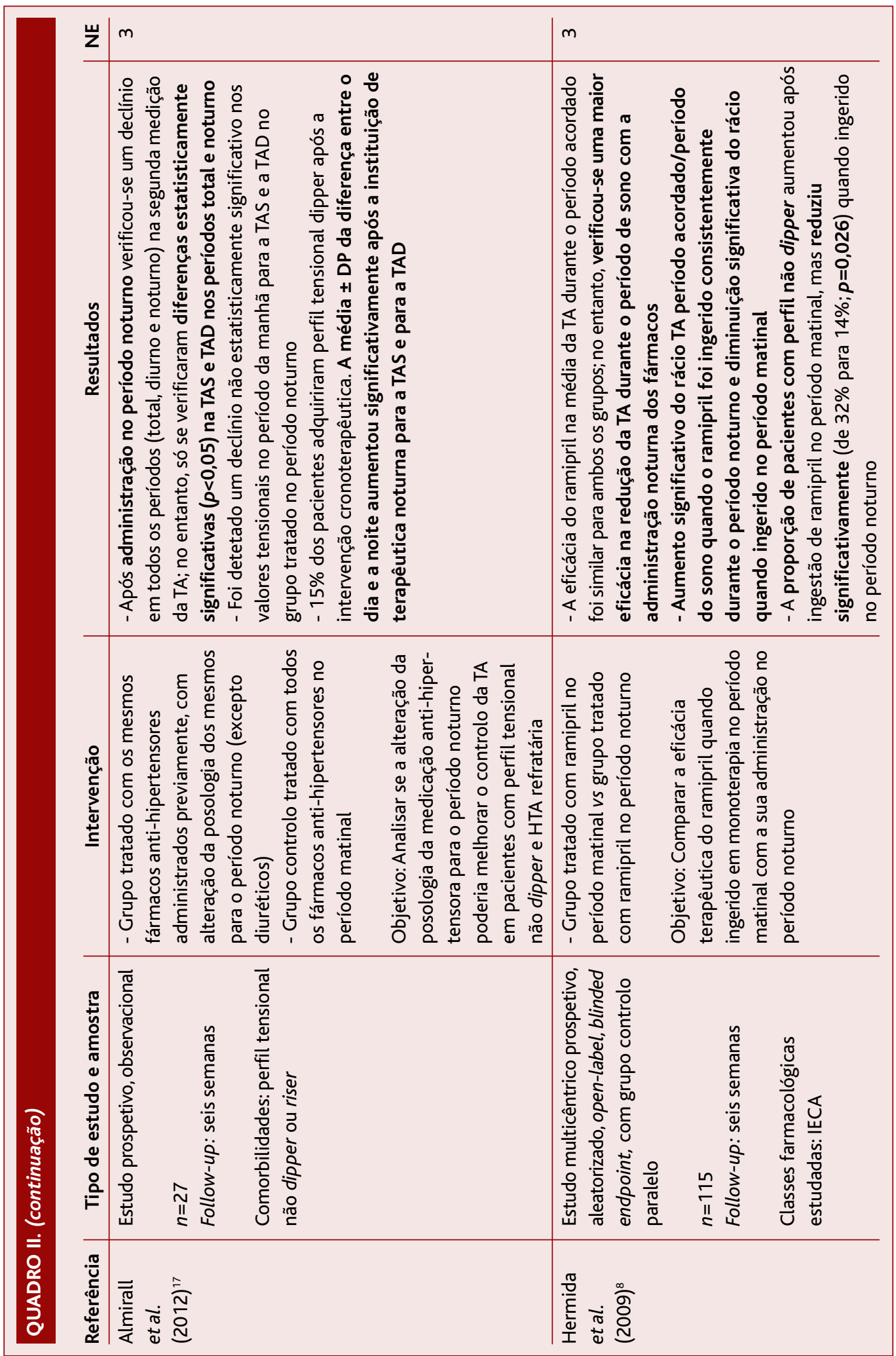




\begin{tabular}{|c|c|c|c|c|c|c|c|c|}
\hline 岂 & $\sim$ & & & & $\sim$ & & & \\
\hline 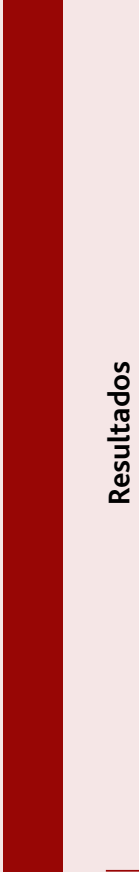 & 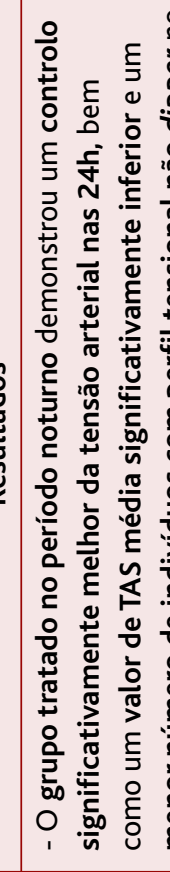 & 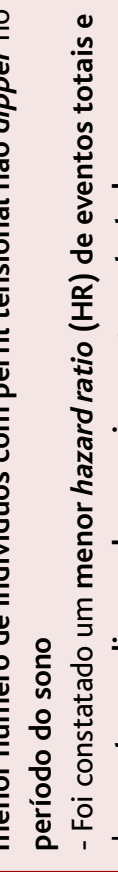 & 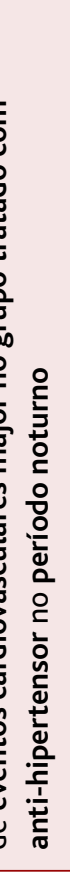 & & 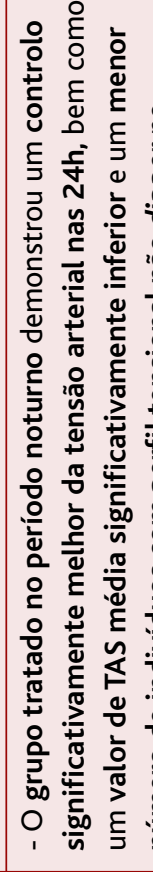 & 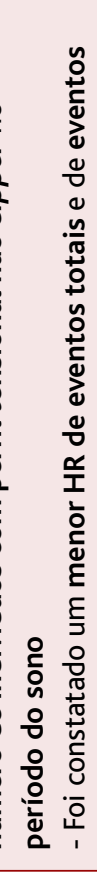 & 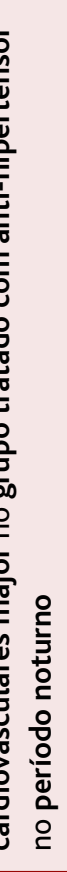 & \\
\hline 惫 & 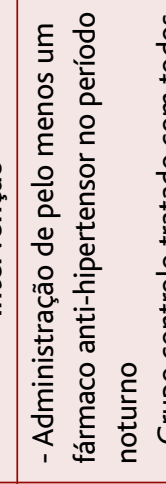 & 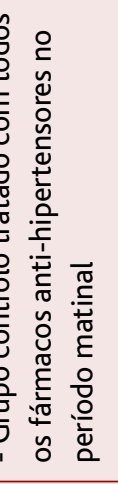 & & 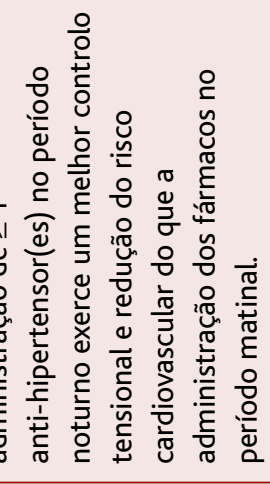 & 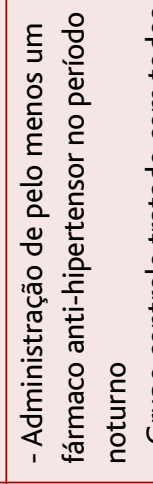 & 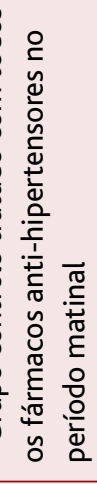 & & 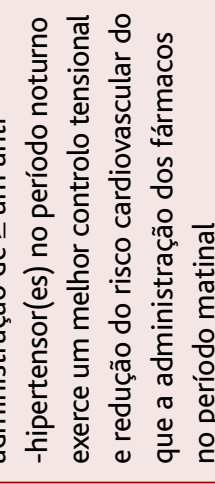 \\
\hline 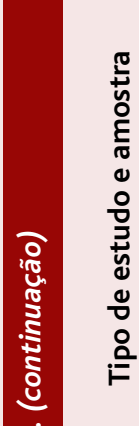 & 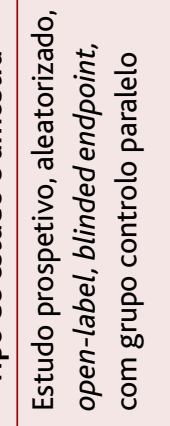 & 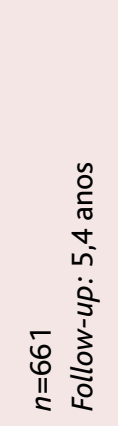 & 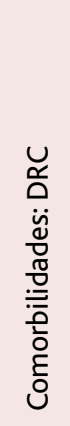 & 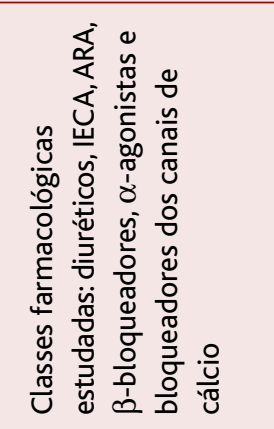 & 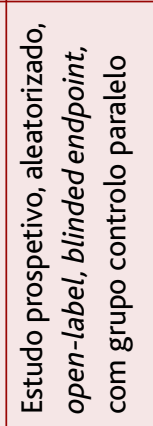 & 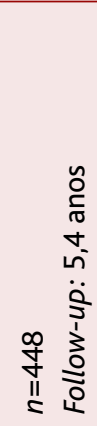 & 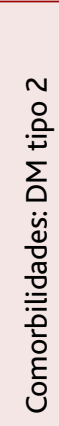 & 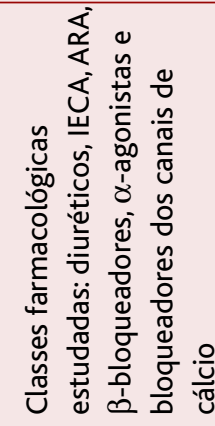 \\
\hline 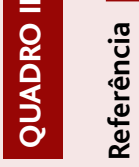 & 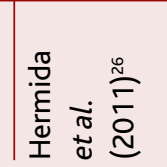 & & & & 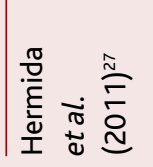 & & & \\
\hline
\end{tabular}


nação de anti-hipertensores (valsartan + amlodipina) no período noturno, o que também foi verificado para o valor da TA no período de sono, com uma queda tensional até um perfil dipper normal. A diferença na eficácia farmacológica foi significativamente superior para a TAS relativamente à TAD, sendo o efeito da administração de ambos os fármacos no período noturno aproximadamente o dobro dos restantes. Destaca-se ainda o facto de o controlo tensional em ambulatório ser significativamente superior nos pacientes tratados com os dois fármacos no período noturno, comparativamente aos grupos submetidos a tratamento com ambos os fármacos no período diurno ou quando um dos fármacos era administrado no período diurno e o outro no período noturno. A administração de ambos os fármacos no período matinal, por sua vez, contribuiu para uma redução da TAD significativamente maior durante a primeira hora de administração face às $12 \mathrm{~h}$ restantes.

Já em 2016, Hermida e colaboradore ${ }^{10}$ realizaram um novo estudo prospetivo e corroboraram os resultados do estudo anterior com a deteção de um menor rácio TAS/TAD durante o sono e uma proporção de pacientes com perfil tensional não dipper significativamente menor no grupo tratado no período noturno, comparativamente ao grupo tratado no período matinal. A proporção de participantes com TA controlada em ambulatório durante o período relativo ao sono foi também significativamente maior neste grupo de utentes e a TAS média nas $48 \mathrm{~h}$ significativamente menor. No entanto, não foram detetadas alterações estatisticamente significativas entre grupos no que respeita aos valores deTAS e TAD no período acordado e na TAD média das $48 \mathrm{~h}$.

Fujiwara e seus colaboradores ${ }^{11}$ realizaram um estudo prospetivo multicêntrico com o intuito de avaliar se a administração matinal do tratamento de combinação valsartan + amlodipina demonstrava inferioridade comparativamente à administração noturna da mesma terapêutica em termos de redução da TA central e da TA braquial noturna. A administração matinal dos fármacos não só se mostrou não inferior à toma noturna, como superior no que respeita à TAS central noturna. Contrariamente, Farah e seus colaboradores ${ }^{12}$ realizaram um estudo com 30 doentes hipertensos tratados com um ou dois fármacos anti-hipertensores no período matinal e valores de TA não controlados. Os doentes foram randomizados em dois grupos: um grupo com administração noturna dos fármacos anti-hipertensores e um grupo controlo com manutenção da administração dos fármacos de manhã. Observou-se que o grupo tratado no período noturno demonstrou uma redução significativa da TA mais proeminente no período noturno e valores tensionais significativamente mais controlados nas $24 \mathrm{~h}$, comparativamente ao grupo tratado no período matinal.

Por sua vez, o estudo de Acelajado e seus colaboradores $^{13}$ evidenciou uma redução significativa da TAS e TAD em todos os períodos estudados (diurno, noturno e 24h) em ambos os grupos de forma semelhante, sem diferenças significativas entre ambos. O número de pacientes com padrão dipper foi superior no grupo tratado com nebivolol no período matinal, sem diferença significativa entre grupos. A única alteração significativa detetada foi a redução da TAS pré-acordar em relação à baseline no grupo tratado no período noturno.

Asmar e seus colaboradores ${ }^{14}$ elaboraram um estudo multicêntrico prospetivo com o intuito de avaliar a eficácia da administração noturna de amlodipina/valsartan, comparativamente à posologia matinal do fármaco em pacientes previamente medicados unicamente com amlodipina $5 \mathrm{mg}$. Objetivaram uma redução significativa da TA noturna no grupo tratado no período noturno, não evidenciada para o período diurno ou $24 \mathrm{~h}$. Em ambos os grupos de tratamento, no entanto, foi notória uma redução significativa da TAS e TAD em todos os períodos do dia.

Os investigadores Hoshino e seus colaboradores ${ }^{15}$ procuraram avaliar a eficácia e segurança da administração de terapêutica combinada com amlodipina e olmesartan no período noturno, tendo realizado um estudo com 31 hipertensos e objetivado uma redução significativamente maior da TAS e TAD às $7 \mathrm{~h}$ neste grupo de doentes comparativamente ao grupo tratado no período matinal. Também foi notória neste grupo uma redução significativa dos valores tensionais no período noturno em indivíduos com perfil não-dipper/riser, o que não foi detetado nos utentes com padrão tensional dipper, e uma TA matinal significativamente menor, com consequente redução do surto matinal de TA.

No estudo de Liu e seus colaboradores ${ }^{16}$ foi verificada uma redução da TAS significativa durante o sono e uma redução ligeira da mesma durante o período acordado nos grupos olmesartan-M (pacientes tratados 
com olmesartan no período matinal) e olmesartan-N (pacientes tratados com olmesartan no período noturno) comparativamente ao grupo valsartan- $\mathrm{N}$ (pacientes tratados com valsartan no período noturno). Ainda, nos grupos olmesartan-M e olmesartan- $\mathrm{N}$, a média da TA diminuiu significativamente durante o período de sono. A redução da TAS no período noturno demonstrou-se superior à redução verificada no período diurno de forma estatisticamente significativa no grupo tratado no período noturno. O número de pacientes com reversão para padrão dipper foi de sete em 11 no grupo valsartan- $\mathrm{N}$, o que corresponde a uma reversão para o padrão dipper em $64 \%$ dos pacientes com passagem do fármaco do período matinal para o período noturno, mantendo a mesma dosagem; ainda, verificou-se uma reversão para padrão dipper de cinco em 11 dos pacientes no grupo olmesartan-M e cinco em 12 dos pacientes no grupo olmesartan-N.

Almirall e seus colaboradores ${ }^{17}$ estudaram 27 utentes com perfil tensional não dipper e HTA refratária, tendo verificado que a passagem da toma dos fármacos para o período noturno produziu um declínio tensional em todos os períodos estudados (total, diurno e noturno); no entanto, as diferenças nos valores de TAS e TAD só foram significativas nos períodos noturno e total. Ainda, quatro dos 27 pacientes adquiriram um perfil tensional dipper após a intervenção cronoterapêutica.

\section{DISCUSSÃO}

A hipertensão arterial é uma doença crónica e um importante fator de risco para o desenvolvimento de doença cardio-cerebrovascular quando não se encontra adequadamente controlada. Por esse motivo, tornase imprescindível a adoção de estilos de vida mais saudáveis e, quando necessário, o seu tratamento adequado e o controlo com fármacos anti-hipertensores.

Alguns artigos postulam que os valores de TA no período noturno constituem um melhor preditor de outcomes do que a TA no período diurno, bem como a presença de um rácio entre os valores tensionais noturnos e diurnos superior a 0,9 , sendo que doentes com perfil tensional não dipper apresentam um risco cardiovascular superior. ${ }^{18}$ Está cientificamente comprovado que pacientes com um perfil tensional noturno não dipper e/ou riser têm um risco acrescido de desenvolvimento de lesão órgão-alvo, doença cardiovascular e/ou mor- talidade global ${ }^{19} \mathrm{e}$ conjetura-se que estes perfis poderão ser revertidos quando a prescrição de anti-hipertensores é efetuada no período noturno e pela administração de diuréticos em indivíduos salt-sensitive. ${ }^{20}$

Por esse motivo, a presente revisão baseada na evidência pretendeu avaliar qual a eficácia a nível do controlo tensional e reversão de perfis não-dippere/ou riser com a administração noturna de anti-hipertensores comparativamente à sua administração convencional (no período matinal). Apenas duas MA e 12 ECA foram elegíveis para a mesma. Os resultados obtidos demonstram que a administração no período noturno de fármacos anti-hipertensores reduz eficazmente os valores de tensão arterial nos períodos diurno, noturno e na média das $24 \mathrm{~h}$ de forma semelhante ao que acontece com a administração destes fármacos no período diurno. No entanto, apenas foram visíveis diferenças significativas entre os dois grupos de tratamento, com superioridade do grupo tratado com anti-hipertensor noturno, na redução dos valores tensionais no período noturno/a dormir.

Apenas quatro dos 14 estudos incluídos na revisão descrevem o efeito da cronoterapia noturna em termos de reversão a perfis dipper, sendo que três corroboram esta teoria ${ }^{8,10,17}$ o o estudo de Liu e colaboradores, apesar de mencionar uma reversão de $64 \%$ com a passagem da toma de valsartan para o período noturno, descreve uma reversão destes perfis em $42-46 \%$ dos utentes com a troca de valsartan por olmesartan nos períodos noturno e matinal. ${ }^{16}$ Este facto poderá dever-se às diferenças já identificadas em estudos com animais anteriores: no estudo verificou-se que a administração de valsartan em períodos inativos reduzia a TA por um maior período de tempo do que quando administrado no período ativo. ${ }^{16}$ Ainda, detetaram que, comparativamente ao valsartan, o olmesartan se dissocia mais lentamente dos recetores AII do tecido vascular, ${ }^{21}$ prolongando desta forma o seu efeito. Este fator torna determinante o papel do médico prescritor na escolha do tipo de anti-hipertensor e da posologia destes fármacos, devendo atender a que utentes com valores tensionais noturnos elevados poderão beneficiar com a administração de anti-hipertensor(es) no período noturno, otimizando assim a farmacodinâmica e farmacocinética destes fármacos. Este aspeto assume particular importância pela premissa de que a relação TA noturna/TA diurna é considerada o fator preditor mais potente para outcomes clínicos, incluindo DCV e mortalidade. ${ }^{22-24}$ 
Um outro aspeto pouco explorado nos artigos selecionados foi a morbimortalidade CV e mortalidade global, que não demonstrou redução com a passagem da administração de fármacos anti-hipertensores para o período noturno nos dois artigos que abordaram esta questão. Ressalva-se que, em ambos os artigos, foi identificado um RR significativamente menor de eventos $\mathrm{CV}$ neste grupo de doentes, corroborando a premissa supracitada.

A presente análise tem como principais limitações a inexistência de homogeneidade de critérios entre estudos, quer em termos da população selecionada (número de participantes, diferentes etnias, utentes com ou sem comorbilidades - DRC, DM - que poderão interferir no controlo tensional) quer do desenho do estudo (duração do follow-up, método e timing de medição dos valores de tensão arterial). Destaca-se ainda a grande diversidade de formas de tratamento e classes de fármacos anti-hipertensores utilizados nos estudos (monoterapia vsterapêutica de combinação, diuréticos $v s$ IECA $v s$ ARA $v s$ BCC $v$ s beta-bloq, diferentes dosagens do mesmo fármaco e diferentes tempos de semivida dos mesmos).

\section{CONCLUSÃO}

Os estudos parecem apontar para uma superioridade na redução dos valores tensionais com a administração dos fármacos anti-hipertensores no período noturno. Este fator torna-se determinante para a cronoterapia dos anti-hipertensores, devendo o médico prescritor atentar ao perfil circadiano tensional de cada utente e tomar uma decisão individualizada acerca do momento de administração mais adequado destes fármacos com o intuito de otimizar a sua eficácia e conseguir ganhos em saúde. No entanto, a avaliação do ritmo circadiano da tensão arterial tem sido negligenciada no processo terapêutico da HTA, pelo que os benefícios que possam advir da normalização dos valores tensionais no período noturno são ainda desconhecidos. São necessários estudos posteriores, com uma maior uniformidade de critérios, dimensão amostral e avaliação da morbimortalidade $\mathrm{CV}$, de forma a poder garantir a eficácia e segurança da administração noturna de fármacos anti-hipertensores e a ponderar a alteração do atual paradigma nacional de posologia matinal destes fármacos.

\section{REFERÊNCIAS BIBLIOGRÁFICAS}

1. Direção-Geral da Saúde. Hipertensão arterial: definição e classificação: norma n. ${ }^{\circ}$ 020/2011, de 28/09/2011, atualizada em 19/03/2013. Lisboa: DGS; 2013.

2. Liu X, Liu X, Huang W, Leo S, Li Y, Liu M, et al. Evening-versus morningdosing drug therapy for chronic kidney disease patients with hypertension: a systematic review. Kidney Blood Press Res. 2014;39(5):427-40.

3. Hermida RC. Ambulatory blood pressure monitoring in the prediction of cardiovascular events and effects of chronotherapy: rationale and design of the MAPEC study. Chronobiol Int. 2007;24(4):749-75.

4. Direção-Geral da Saúde. Abordagem terapêutica da hipertensão arterial: norma n. ${ }^{\circ}$ 026/2011, de 29/09/2011, atualizada em 19/03/2013. Lisboa: DGS; 2013.

5. Zhao $P, X u P$, Wan $C$, Wang Z. Evening versus morning dosing regimen drug therapy for hypertension. Cochrane Database Syst Rev. 2011;(10):CD004184.

6. Wang C, Qiu X, Lv L, Huang J, Li S, Lou T, et al. Chronotherapy for hypertension in patients with chronic kidney disease: a systematic review and meta-analysis in non-black patients. Int Urol Nephrol. 2017;49(4):651-9.

7. Hermida RC, Ayala DE, Fontao MJ, Mojón A, Fernández JR. Chronotherapy with valsartan/amlodipine fixed combination: improved blood pressure control of essential hypertension with bedtime dosing. Chronobiol Int. 2010;27(6):1287-303.

8. Hermida RC, Ayala DE. Chronotherapy with the angiotensin-converting enzyme inhibitor ramipril in essential hypertension. Hypertension. 2009;54(1):40-6.

9. Lacourcière $Y$, Neutel JM, Davidai G, Koval S. A multicenter, 14-week study of telmisartan and ramipril in patients with mild-to-moderate hypertension using ambulatory blood pressure monitoring. Am J Hypertens. 2006;19(1):104-12.

10. Hermida RC,Ayala DE, Mojón A, Fernández JR. Bedtime ingestion of hypertension medications reduces the risk of new-onset type 2 diabetes : a randomised controlled trial. Diabetologia. 2016;59(2):255-65.

11. Fujiwara T, Hoshide S, Yano Y, Kanegae H, Kario K. Comparison of morning vs bedtime administration of the combination of valsartan/amlodipine on nocturnal brachial and central blood pressure in patients with hypertension. J Clin Hypertens. 2017;13(12):1319-26.

12. Farah R, Makhoul N, Arraf Z, Khamisy-Farah R. Switching therapy to bedtime for uncontrolled hypertension with a nondipping pattern: a prospective randomized-controlled study. Blood Press Monit. 2013;18(4):227-31.

13. Acelajado MC, Pisoni R, Dudenbostel T, Oparil S, Calhoun DA, Glasser SP. Both morning and evening dosing of nebivolol reduces trough mean blood pressure surge in hypertensive patients. J Am Soc Hypertens. 2012;6(1):66-72.

14. Asmar R, Gosse P, Queré S, Achouba A. Efficacy of morning and evening dosing of amlodipine / valsartan combination in hypertensive patients uncontrolled by $5 \mathrm{mg}$ of amlodipine phane Quere. Blood Press Monit. 2011;16(2):80-6.

15. Hoshino A, Nakamura T, Matsubara $\mathrm{H}$. The bedtime administration ameliorates blood pressure variability and reduces urinary albumin excretion in amlodipine-olmesartan combination therapy. Clin Exp Hypertens. 2010;32(7):416-22.

16. Liu Y, Ushijima K, Ohmori M, Takada M, Tateishi M, Ando H, et al. Chronopharmacology of angiotensin II-receptor blockers in stroke-prone 
spontaneously hypertensive rats. J Pharmacol Sci. 2011;115(2):196-204. 17. Almirall J, Comas L, Martínez-Ocaña JC, Roca S, Arnau A. Effects of chronotherapy on blood pressure control in non-dipper patients with refractory hypertension. Nephrol Dial Transpl. 2012;27(5):1855-9.

18. Williams B, Mancia G, Spiering W, Rosei EG, Azizi M, Burnier M, et al. 2018 ESC/ESH Guidelines for the managment of arterial hypertension: the Task Force for the management of arterial hypertension of the European Society of Cardiology (ESC) and the European Society of Hypertension (ESH). Eur Heart J. 2018;39(33):3021-104.

19. Bankir L, Bochud M, Maillard M, Bovet P, Gabriel A, Burnier M. Nighttime blood pressure and nocturnal dipping are associated with daytime urinary sodium excretion in African subjects. Hypertension. 2008;51(4):891-8.

20. Cuspidi C, Giudici V, Negri F, Sala C. Nocturnal nondipping and left ventricular hypertrophy in hypertension: an updated review. Expert Rev Cardiovasc Ther. 2010;8(6):781-92.

21. Ushijima K, Nakashima H, Shiga T, Harada K, Ishikawa S, loka T, et al. Different chronotherapeutic effects of valsartan and olmesartan in non-dipper hypertensive patients during valsartan treatment at morning. J Pharmacol Sci. 2015;127(1):62-8.

22. Muxfeldt ES, Cardoso CR, Salles GF. Prognostic value of nocturnal blood pressure reduction in resistant hypertension. Arch Intern Med. 2009;169(9):874-80.

23. Dolan E, Stanton A, Thijs L, Hinedi K, Atkins N, McClory S, et al. Superiority of ambulatory over clinic blood pressure measurement in predicting mortality: the Dublin outcome study. Hypertension. 2005;46(1):156-61.

24. Staessen JA, Thijs L, Fagard R, O'Brien ET, Clement D, de Leeuw PW, et al. Predicting cardiovascular risk using conventional vs ambulatory blood pressure in older patients with systolic hypertension. JAMA. 1999;282(6):539-46.

25. Ushijima K, Nakashima H, Shiga T, Harada K, Ishikawa S, loka T, et al. Different chronotherapeutic effects of valsartan and olmesartan in non-dipper hypertensive patients during valsartan treatment at morning. J Pharmacol Sci. 2015;127(1):62-8.

26. Hermida RC, Ayala DE, Mojo A, Fernández JR. Bedtime dosing of antihypertensive medications reduces cardiovascular risk in CKD. J Am Soc Nephrol. 2011;22(12):2313-21.

27. Hermida RC, Ayala D, Mojón A, Fernandéz J. Influence of time of day of blood pressure: lowering treatment on patients with type 2 diabetes. Diabetes Care. 2011;34(6):1270-6.

\section{CONFLITO DE INTERESSES}

As autoras declaram não ter quaisquer conflitos de interesse.

\section{FINANCIAMENTO}

As autoras declaram não ter recebido subsídios ou bolsas para a elaboração do artigo.

\author{
ENDEREÇO PARA CORRESPONDÊNCIA \\ Ângela Oliveira Mendes \\ E-mail: asomendes@gmail.com \\ https://orcid.org/0000-0003-1016-5581
}

Recebido em 31-05-2019

Aceite para publicação em 03-01-2020

\section{ABSTRACT}

\section{CHRONOTHERAPY OF ANTIHYPERTENSIVE DRUGS: AN EVIDENCE-BASED REVIEW}

Objective: To determine whether nocturnal administration of antihypertensive drugs may have a benefit in terms of efficacy in reducing blood pressure and/or cardiovascular events, morbidity, and mortality compared to morning administration.

Data sources: National Guideline Clearinghouse, NHS Evidence, CMA Infobase, The Cochrane Library, DARE and MEDLINE/PubMed.

Methods: Search of guidelines, systematic reviews (SR), meta-analysis (MA) and clinical trials published between January 2008 and October 2018, available in English and Portuguese under the terms MeSH 'Antihypertensive Agents' and 'Drug Chronotherapy'. The American Family Physician's Strength of Recommendation Taxonomy (SORT) was used to assess the levels of evidence (NE) and the strength of recommendation (FR).

Results: Fifty-five articles were obtained in the initial research, of which 13 fulfilled the inclusion criteria. With nocturnal administration of drugs, greater control of blood pressure values at night was observed, and the reversion of dipper profiles to non-dipper, with no statistically significant differences in the diurnal period. Only MA analyzed global and cardiovascular mortalities, and no statistically significant changes were detected in this parameter between the morning and evening groups; however, a lower relative risk of cardiovascular events in the evening group was evident in three of the studies.

Conclusion: The administration of antihypertensive drugs in the nocturnal period proved to have benefit in terms of reduction of blood pressure values and showed superiority in this reduction during nocturnal period, allowing even to revert non-dipper/riser patterns to the dipper. However, further studies, with greater uniformity of criteria and evaluation of CV morbidity and mortality, are required in order to ensure the efficacy and safety of nocturnal administration of antihypertensive drugs.

Keywords: Chronotherapy; Antihypertensive drugs. 